\title{
Job Performance of Medical Graduates with Compulsory Services in Underserved Rural Areas in China: A Cohort Study
}

\section{Mingyue Li}

Peking University School of Public Health https://orcid.org/0000-0001-8417-5665

\section{Ziyue Wang}

Peking University Health Science Centre

\section{Baisong Zhang}

Peking University School of Public Health

\section{Tiantian Wei}

Peking University School of Public Health

\section{Dan $\mathrm{Hu}$}

Peking University Health Science Centre

Xiaoyun Liu ( $\nabla$ xiaoyunliu@pku.edu.cn )

Peking University Health Science Centre https://orcid.org/0000-0002-5483-0742

\section{Research}

Keywords: compulsory services programs, rural health service, human resources for health, job performance

Posted Date: May 5th, 2021

DOI: https://doi.org/10.21203/rs.3.rs-472206/v1

License: (9) This work is licensed under a Creative Commons Attribution 4.0 International License.

Read Full License

Version of Record: A version of this preprint was published at International Journal of Health Policy and Management on January 19th, 2022. See the published version at https://doi.org/10.34172/ijhpm.2022.6335. 


\section{Abstract}

\section{Objective}

China started a national program in 2010 to train qualified general practitioners with compulsory services in rural and remote areas. While this compulsory services program (CSP) has shown positive effects on staffing primary health care in rural areas, very little is known about how well they perform in the rural service. This study aims to evaluate the job performance of those medical graduates from this program and how the program design has influenced their job performance.

\section{Methods}

A cohort study was conducted with medical graduates from CSP and non-CSP (NCSP) in four medical universities in central and western China. Baseline survey and three waves of follow-up surveys were conducted from 2015-2020. The pass rate of China National Medical Licensing Examinations (NMLE) and self-reported job performance were used as measurements of job performance. Comparisons were made between CSP and NCSP graduates. Multivariable regressions were used to identify factors affecting job performance.

\section{Results}

2154 medical graduates were included in the sample, with 1586 CSP graduates and 568 NCSP graduates. CSP (90.6\%) and NCSP (87.5\%) graduates showed no difference in passing the NMLE $(P=0.153)$. CSP graduates reported high job performance, similar to those NCSP graduates (CSP, 63.7; NCSP, 64.2). In the design of CSP, having officially budget posts improved job performance of CSP ( $\beta$ coefficient=4.87, $P \llbracket 0.05$ ). Working in hometown was also a significant factor that increase job performance when controlling the differences in contracting strategy ( $\beta$ coefficient=1.48, $P \llbracket 0.05)$.

\section{Conclusion}

Medical students trained by CSP have demonstrated good job performance in primary healthcare systems in China. Compared to NCSP peers who work in urban areas in majority, CSP graduates' high job performance could increase the accessibility of equal- or high-quality services in rural areas, which contributes to qualified workforce supply and health equity. Providing officially budgeted posts and working in hometowns can affect job performance and future retention. Early career support can have critical importance for CSP graduates.

\section{Introduction}

One of the most challenging tasks of policy-makers is to provide people living in rural and remote areas access to affordable care from well-trained health workers [1-3]. This problem is common to almost all countries and poses a major challenge to the nationwide provision of equitable health services [4-6]. In 2010, the World Health Organization issued global policy recommendations focusing on increasing 
access to health workers in remote and rural areas [1]. Countries around the world have explored and implemented a variety of interventions to train, attract, and retain health workers for rural areas [7-13], including compulsory services programs (CSP) [14-16]. Since the early 20th century, CSP have been implemented in many countries [14], and are deemed a way of pursing social justice and health equity because they enable governments to allocate health resources to areas that are scarce in resources and to communities that are discarded by market forces. According to Dolea et. al. [17], very little is known about the effectiveness of CSP. High job performance is crucial for health workers to provide quality care, thereby strengthening health systems and improving health outcomes $[18,19]$. If CSP can improve health worker supply for rural areas but with inferior job performance and service quality, the concerns about health inequity between rural and urban areas will remain a critical issue. However, most evaluations focused on attraction and retention of health workers for rural areas [20-22], few, if any, pay attention to how those retained health workers perform in rural areas [23].

China's primary health system (PHC), which provides basic care and public health services to 1.4 billion population, faces great challenges in its capacity and equity [24]. Inadequate qualified health workers and insufficient competence of current primary health workforce impede access to appropriate healthcare services. Healthcare services provided by PHC cannot meet people's need and patients bypass PHC in favor of costly tertiary hospitals. From 2010 to 2019, the percentage of outpatient and inpatients visits of primary healthcare facilities to all healthcare facilities has decreased year by year [25]. In rural and remote areas, this problem is even more significant because more health workers are concentrated in urban and wealthier areas.

In order to enhance the capability of rural primary healthcare and increase health accessibility and equity, in 2010, China started a national CSP for medical students, training general practitioners for rural areas in central and western regions [26]. Medical universities and colleges recruit students with rural backgrounds. When admitted to the CSP, students usually had a slightly lower performance in the university entrance examination than their NCSP peers. The medical education curriculum is similar for the two programs, except that the CSP students have more rural exposures during the internship period. On matriculation, students need to sign contracts with local health administrations and medical universities, and commit to go to the appointed township health centers or village clinics to practice for six years soon after five-year undergraduate medical education. Students do not need to pay for tuition or accommodation, and can receive monthly allowances. The county with which the CSP graduates are required to sign contract are decided by local government depending on the local demand and supply of health workforce. Preliminary evaluations have shown that the majority of medical graduates in this program can fulfill the contract to work in a rural area [27-29]. It has been five years since the first wave of students graduating from compulsory services program. More than 5000 medical students graduate from this program each year in China. Much still needs to be understood about their competency and how they perform in rural and remote areas and how the design of CSP have influenced their performance. 
In this paper, we examined the job performance of medical students graduating from CSP in four medical universities in China, and compared with their school peers (NCSP). This study can take us a step further in understanding the implementation and the effect of recruiting and retaining health workers for underserved areas through CSP, and provides evidence for other countries that are also seeking to increase access to health workers in underserved areas.

\section{Methods}

\section{Data sources}

Data used in this paper were retrieved from Cohort Study of Medical Graduates with Compulsory Services in Rural Areas Studies, funded by China Medical Board (CMB). The survey is approved by the institutional review board (IRB) at Peking University Health Science Center (IRB00001052-15027). The study was launched in 2015 and has established five cohorts in five years of medical graduates. The cohorts have been defined by their year of graduation. We collected baseline data before students graduate and follow-up data every year after their graduation. Till now, we have collected follow-up data in 2016, 2017, 2018, and 2020. Jiangxi, Qinghai and Guangxi provinces were chosen from western and central China, representing middle- and low-level economic regions. The survey included 3620 medical graduates from Medical college of Qinghai University (Qinghai province), Jiujiang University (Jiangxi province), Gannan Medical University (Jiangxi province), and Guangxi Medical Universities (Guangxi Zhuang autonomous region). The location of three provinces and four universities in China is presented in Figure 1, with the initial cohort size.

There are two types of medical graduates in this study, those who are required to practice in rural and remote areas after graduation (Compulsory services program, CSP) as the intervention group, and common medical graduates (non-compulsory services program, NCSP) as the comparison group. We collected information on demographic characteristics, employment, postgraduate study, residency training and job-related information for the two types of graduates.

\section{Methods of measurement}

\section{Pass rate of National Medical Licensing Examinations (NMLE)}

In China, the NMLE has been implemented for 20 years since the 1999 Law on Practicing Doctors [30]. The NMLE is sponsored by China National Medical Examination Center, an affiliated institution of National Health Commission. All practicing doctors in China must pass the NMLE before they can register as a licensed doctor or a licensed assistant doctor in order to practice legally. The NMLE includes clinical skill test and general medical knowledge test, which can well reflect candidates' professional 
competency [31]. We adopted the pass rate of NMLE as an objective indicator of CSP graduates' job performance, and compared with that of NCSP graduates.

\section{Job performance scale}

Conceptual grounds for job performance can be found in well-established theories of human capital and psychology [32]. Job performance was defined as the "aggregated value to the organization of discrete behavior episodes that an individual performs over a standard interval of time" [33]. To our knowledge, job performance is generally measured from two dimensions, including task performance and contextual performance. Measuring job performance by scale has been proved reliable and consistent compared with results from that measured by objective indicators [34]. Most scales were designed from task and contextual dimensions before. However, learning capability for employees has been attached to more importance in job performance studies, and recent scales have included this dimension $[35,36]$. The scale we adopted included measurements of all three dimensions of job performance [37]. This scale was based upon widely-used international job performance scale and revised in adaptation to Chinese context. It has been used in studies on rural health workers and GPs' job performance and has proved valid cultural adaptation [38-40].

The self-reported job performance scale included three dimensions and 12 items (task performance: 4 items; contextual performance: 5 items; learning performance: 3 items). The definition for each dimension is presented in Table 1. The items are rated on a 7-point Likert scale of how often the behavior or feeling, or attitude has been manifested: $1=$ never, $2=a$ few times a year or less, $3=$ once a month, $4=a$ few times a year, and $5=$ once a week, $6=a$ few times a week; $7=$ every day. The total score ranges from 1 (lowest job performance) to 84 (highest job performance). One's total job performance score was obtained by adding the scores of each item. Score for each dimension was also calculated. The alpha internal consistency coefficient of reliability was 0.89 in this study, indicating that the items were highly internally consistent. The validity was examined through incremental fit index (IFI) and showed good validity [37].

Table 1

Dimensions, definitions, and reliability of the job performance scale 


\begin{tabular}{|c|c|c|c|}
\hline Dimensions & Definition & $\begin{array}{l}\text { Cronbach's } \\
\text { a }\end{array}$ & $\begin{array}{l}\text { Cronbach's } \\
\text { a in } \\
\text { literature }\end{array}$ \\
\hline $\begin{array}{l}\text { Task } \\
\text { performance }\end{array}$ & $\begin{array}{l}\text { Related to work output and directly serving } \\
\text { organizational goals } \varangle \text { reflected in work quality, efficiency, } \\
\text { ability and input }\end{array}$ & 0.947 & 0.805 \\
\hline $\begin{array}{l}\text { Learning } \\
\text { performance }\end{array}$ & $\begin{array}{l}\text { The process that individuals form learning plans and } \\
\text { apply new knowledge and skills to the changing } \\
\text { organizational environment, including learning desires, } \\
\text { behavior and result application }\end{array}$ & 0.954 & 0.842 \\
\hline $\begin{array}{l}\text { Contextual } \\
\text { performance }\end{array}$ & $\begin{array}{l}\text { Indirect assistance to the realization of organizational } \\
\text { goals, efforts to improve the harmony of working } \\
\text { environment, including assisting colleagues, abiding by } \\
\text { rules, extra efforts, personal self-discipline, supporting } \\
\text { organizational goals, etc. }\end{array}$ & 0.936 & 0.714 \\
\hline Total & - & 0.897 & - \\
\hline
\end{tabular}

\section{Statistical analysis}

Descriptive analysis was used to identify characteristics of the sample. Percentages were calculated for qualitative data, and means and standard deviations (SD) for quantitative data. For pass rate of NMLE, Chi-square test was used to compare the differences between CSP and NCSP graduates. For job performance scale, we determined the total scores of job performance scale for CSP and NCSP graduates separately by different demographic and job-related variables. One-way analysis of variance (ANOVA) was used to examine the differences among these characteristics. The P-value below 0.05 was considered statistically significantly.

The job-related factors, including obtaining officially budgeted posts (which means these are tenure positions with full salary support, secured pensions and other benefits from government budget, and are considered as formal employees of Chinese public sectors) becoming attending physicians, working in hometown district, all showed significant difference for CSP graduates in ANOVA, so we further fitted multivariable linear regression models to explore the association between the design of the program and job performance for CSP graduates. Total job performance scores were used as the dependent variable. We also conducted robustness check to verify our findings. Firstly, we used logarithm of total performance score for regression, with coefficients and standard errors reported. Secondly, a dichotomous indicator of job performance variable was used for logistic regression (those obtaining 6 or 7 for each item with the total score being or above 72 were identified as high job performance and was coded as " 1 ", otherwise coded as " 0 "). Odds ratios (OR) and corresponding 95\% confidence intervals (Cl) were reported (see Appendix). Lastly, the three sub-dimensions of job performance, score of task, 
learning, and contextual performance were replaced as the dependent variables for regression (see Appendix). All statistical analyses were conducted in Stata 15.1 (Stata Corp LP, College Station).

\section{Results}

\section{Basic characteristics of the cohort}

Around 300-400 students were included in each baseline survey from 2015 to 2019 in the five cohorts. The response rate was higher for CSP than NCSP across cohorts (Table 2). The sample used for analysis in this study were limited to those who are currently working, otherwise they cannot answer the job performance scale, for example, those who are undertaking postgraduate study. Table 3 shows the social demographic characteristics of the sample. The total sample amounts to 2154 , including 1586 CSP, accounting for $73.6 \%$ (Table 3). The majority of CSP (97.5\%) have obtained officially budgeted posts, while only $33.6 \%$ of NCSP have got the officially budgeted posts. The average monthly income of graduates of CSP (3883 CNY per month) is $34.2 \%$ lower than that of NCSP (5903 CNY per month). Most graduates of CSP work in the contract signing places (97.8\%), but only $59.6 \%$ of them work in their hometown. Although they have graduated 5 years at most, some graduates of CSP (7.1\%) have been promoted to attending physicians.

Table 2

The response rate for CSP and NCSP graduates in 2020 wave

\begin{tabular}{|lllllll|}
\hline Years of & CSP & \multicolumn{5}{c|}{ NCSP } \\
\cline { 2 - 7 } graduation & Baseline & $\mathbf{2 0 2 0}$ wave & Response rate & Baseline & $\mathbf{2 0 2 0}$ wave & Response rate \\
& $\mathbf{N}$ & $\mathbf{N}$ & $\%$ & $\mathbf{N}$ & $\mathbf{N}$ & $\%$ \\
\hline 2015 & 305 & 239 & $78.4 \%$ & 315 & 181 & $57.5 \%$ \\
\hline 2016 & 437 & 330 & $75.5 \%$ & 302 & 163 & $54.0 \%$ \\
\hline 2017 & 481 & 383 & $79.6 \%$ & 330 & 203 & $61.5 \%$ \\
\hline 2018 & 437 & 328 & $75.1 \%$ & 312 & 201 & $64.4 \%$ \\
\hline 2019 & 381 & 351 & $92.1 \%$ & 320 & 261 & $81.6 \%$ \\
\hline $\begin{array}{l}\text { Note: The 2020 wave is the fourth, the third, the second and the first follow-up for 2015, 2016, 2017, } \\
\text { 2018, and 2019 graduates, respectively. }\end{array}$
\end{tabular}


Table 3

Social demographic characteristics of CSP and NCSP graduates, $n(\%)$

\begin{tabular}{|c|c|c|c|c|}
\hline Characteristics & Variables & $\begin{array}{l}\operatorname{CSP}(n= \\
1586)\end{array}$ & $\begin{array}{l}\operatorname{NCSP}(n= \\
568)\end{array}$ & $\begin{array}{l}\text { Overall }(n= \\
2154)\end{array}$ \\
\hline \multirow[t]{4}{*}{ School } & Qinghai & $\begin{array}{l}504 \\
(31.78 \%)\end{array}$ & $\begin{array}{l}166 \\
(29.23 \%)\end{array}$ & $\begin{array}{l}670 \\
(31.10 \%)\end{array}$ \\
\hline & Guangxi & $\begin{array}{l}408 \\
(25.73 \%)\end{array}$ & $\begin{array}{l}173 \\
(30.46 \%)\end{array}$ & $\begin{array}{l}581 \\
(26.97 \%)\end{array}$ \\
\hline & Jiujiang & $\begin{array}{l}181 \\
(11.41 \%)\end{array}$ & $\begin{array}{l}117 \\
(20.60 \%)\end{array}$ & $\begin{array}{l}298 \\
(13.83 \%)\end{array}$ \\
\hline & Gannan & $\begin{array}{l}493 \\
(31.08 \%)\end{array}$ & $\begin{array}{l}112 \\
(19.72 \%)\end{array}$ & $\begin{array}{l}605 \\
(28.09 \%)\end{array}$ \\
\hline \multirow[t]{5}{*}{ Year of graduation } & 2015 & $\begin{array}{l}219 \\
(13.81 \%)\end{array}$ & $\begin{array}{l}152 \\
(26.76 \%)\end{array}$ & $\begin{array}{l}371 \\
(17.22 \%)\end{array}$ \\
\hline & 2016 & $\begin{array}{l}326 \\
(20.55 \%)\end{array}$ & $\begin{array}{l}126 \\
(22.18 \%)\end{array}$ & $\begin{array}{l}452 \\
(20.98 \%)\end{array}$ \\
\hline & 2017 & $\begin{array}{l}377 \\
(23.77 \%)\end{array}$ & $\begin{array}{l}122 \\
(21.48 \%)\end{array}$ & $\begin{array}{l}499 \\
(23.17 \%)\end{array}$ \\
\hline & 2018 & $\begin{array}{l}317 \\
(19.99 \%)\end{array}$ & $\begin{array}{l}90 \\
(15.85 \%)\end{array}$ & $\begin{array}{l}407 \\
(18.90 \%)\end{array}$ \\
\hline & 2019 & $\begin{array}{l}347 \\
(21.88 \%)\end{array}$ & $\begin{array}{l}78 \\
(13.73 \%)\end{array}$ & $\begin{array}{l}425 \\
(19.73 \%)\end{array}$ \\
\hline \multirow[t]{4}{*}{$\begin{array}{l}\text { Demographic } \\
\text { characteristics }\end{array}$} & Female & $\begin{array}{l}762 \\
(48.05 \%)\end{array}$ & $\begin{array}{l}294 \\
(51.76 \%)\end{array}$ & $\begin{array}{l}1056 \\
(49.03 \%)\end{array}$ \\
\hline & Male & $\begin{array}{l}824 \\
(51.95 \%)\end{array}$ & $\begin{array}{l}274 \\
(48.24 \%)\end{array}$ & $\begin{array}{l}1098 \\
(50.97 \%)\end{array}$ \\
\hline & Married & $\begin{array}{l}498 \\
(31.40 \%)\end{array}$ & $\begin{array}{l}178 \\
(31.34 \%)\end{array}$ & $\begin{array}{l}676 \\
(31.38 \%)\end{array}$ \\
\hline & Not married & $\begin{array}{l}1088 \\
(68.60 \%)\end{array}$ & $\begin{array}{l}390 \\
(68.66 \%)\end{array}$ & $\begin{array}{l}1478 \\
(68.62 \%)\end{array}$ \\
\hline \multirow[t]{3}{*}{ Current workplace } & $\begin{array}{l}\text { Public hospitals at county level } \\
\text { and above }\end{array}$ & $\begin{array}{l}131 \\
(8.26 \%)\end{array}$ & $\begin{array}{l}510 \\
(89.79 \%)\end{array}$ & $\begin{array}{l}641 \\
(29.76 \%)\end{array}$ \\
\hline & $\mathrm{CHC} \& \mathrm{THC}$ & $\begin{array}{l}1446 \\
(91.17 \%)\end{array}$ & $12(2.11 \%)$ & $\begin{array}{l}1458 \\
(67.69 \%)\end{array}$ \\
\hline & Other & $9(0.57 \%)$ & $46(8.10 \%)$ & $55(2.55 \%)$ \\
\hline $\begin{array}{l}\text { Current job } \\
\text { Information }\end{array}$ & $\begin{array}{l}\text { Obtaining officially budgeted } \\
\text { posts }\end{array}$ & $\begin{array}{l}1546 \\
(97.48 \%)\end{array}$ & $\begin{array}{l}191 \\
(33.63 \%)\end{array}$ & $\begin{array}{l}1737 \\
(80.64 \%)\end{array}$ \\
\hline
\end{tabular}




\begin{tabular}{|c|c|c|c|c|}
\hline Characteristics & Variables & $\begin{array}{l}\operatorname{CSP}(n= \\
1586)\end{array}$ & $\begin{array}{l}\operatorname{NCSP}(n= \\
568)\end{array}$ & $\begin{array}{l}\text { Overall }(n= \\
2154)\end{array}$ \\
\hline & $\begin{array}{l}\text { Income of current job /month } \\
\text { (mean } \pm \text { SD) }\end{array}$ & $\begin{array}{l}3883 \pm \\
3379\end{array}$ & $\begin{array}{l}5903 \pm \\
5397\end{array}$ & $4416 \pm 4107$ \\
\hline & $\begin{array}{l}\text { Working in contract signing } \\
\text { place }\end{array}$ & $\begin{array}{l}1551 \\
(97.79 \%)\end{array}$ & - & $\begin{array}{l}1551 \\
(72.01 \%)\end{array}$ \\
\hline & Working in hometown district & $\begin{array}{l}945 \\
(59.58 \%)\end{array}$ & - & $\begin{array}{l}945 \\
(43.87 \%)\end{array}$ \\
\hline & Becoming Attending physicians & $\begin{array}{l}113 \\
(7.12 \%)\end{array}$ & $2(0.35 \%)$ & $115(5.34 \%)$ \\
\hline
\end{tabular}

\section{Results of pass rate of China National Medical Licensing Examinations}

Overall, CSP graduates showed no difference in the NMLE compared with NCSP graduates $(P=0.153)$. In Qinghai, Guangxi, and Jiujiang, CSP graduates all reported higher rates of passing the examination than their counterparts, and did not show statistically significant difference with NCSP graduates. In Gannan, however, CSP graduates reported a significantly higher rate of passing the examination $(P=0.002)$.

Table 4

Pass rate of the China NMLE for CSP and NCSP graduates

\begin{tabular}{|c|c|c|c|c|c|c|}
\hline \multirow[t]{2}{*}{ Schools } & \multicolumn{2}{|l|}{ CSP } & \multicolumn{2}{|c|}{ NCSP } & \multirow[t]{2}{*}{ Overall } & \multirow[t]{2}{*}{$P$ value } \\
\hline & $\mathbf{N}$ & $\%$ & $\mathbf{N}$ & $\%$ & & \\
\hline Qinghai & 268 & $76.8 \%$ & 101 & $76.5 \%$ & $76.7 \%$ & 0.949 \\
\hline Guangxi & 312 & $98.4 \%$ & 148 & $96.7 \%$ & $97.9 \%$ & 0.234 \\
\hline Jiujiang & 148 & $93.7 \%$ & 89 & $89.0 \%$ & $91.9 \%$ & 0.181 \\
\hline Gannan & 388 & $95.1 \%$ & 83 & $86.5 \%$ & $93.5 \%$ & 0.002 \\
\hline Overall & 1116 & $90.6 \%$ & 421 & $87.5 \%$ & $89.7 \%$ & 0.153 \\
\hline
\end{tabular}

\section{Results of job performance scale}


Table 5 displays the results of their job performance measured by 7-point Likert scale. The total job performance score of CSP (21.12) is basically the same with NCSP (21.50). The difference in task performance, contextual performance, and learning performance are also small between graduates of CSP and NCSP. Both graduates of CSP and NCSP show higher scores on the item "I can always strictly abide by the rules and regulations of the institution" in contextual performance. They exhibit lower scores on the item "I will provide some good suggestions of the management decisions for employers" in contextual performance. 
Table 5

Mean score (SD) of each item in the 7-point Likert job performance scale

\begin{tabular}{|c|c|c|c|}
\hline Dimensions & Items & CSP & NCSP \\
\hline \multirow[t]{5}{*}{$\begin{array}{l}\text { Task } \\
\text { performance }\end{array}$} & Total & $\begin{array}{l}21.12 \\
(4.35)\end{array}$ & $\begin{array}{l}21.50 \\
(3.96)\end{array}$ \\
\hline & I can always finish my work in time skillfully & $\begin{array}{l}5.38 \\
(1.20)\end{array}$ & $\begin{array}{l}5.46 \\
(1.12)\end{array}$ \\
\hline & $\begin{array}{l}\text { I can always finish my work with high quality according to the } \\
\text { requirements of performance appraisal }\end{array}$ & $\begin{array}{l}5.31 \\
(1.23)\end{array}$ & $\begin{array}{l}5.42 \\
(1.08)\end{array}$ \\
\hline & I have very good job-related knowledge and skills & $\begin{array}{l}5.23 \\
(1.22)\end{array}$ & $\begin{array}{l}5.25 \\
(1.08)\end{array}$ \\
\hline & I always put all my energy into my work & $\begin{array}{l}5.20 \\
(1.26)\end{array}$ & $\begin{array}{l}5.37 \\
(1.15)\end{array}$ \\
\hline \multirow[t]{6}{*}{$\begin{array}{l}\text { Contextual } \\
\text { performance }\end{array}$} & Total & $\begin{array}{l}26.45 \\
(5.32)\end{array}$ & $\begin{array}{l}26.61 \\
(4.81)\end{array}$ \\
\hline & I often offer help to other colleagues & $\begin{array}{l}5.39 \\
(1.22)\end{array}$ & $\begin{array}{l}5.51 \\
(1.12)\end{array}$ \\
\hline & $\begin{array}{l}\text { I can always strictly abide by the rules and regulations of the } \\
\text { institution }\end{array}$ & $\begin{array}{l}6.04 \\
(1.13)\end{array}$ & $\begin{array}{l}6.04 \\
(1.05)\end{array}$ \\
\hline & $\begin{array}{l}\text { Even without the supervision of the leader, I can always work } \\
\text { with enthusiasm }\end{array}$ & $\begin{array}{l}5.51 \\
(1.26)\end{array}$ & $\begin{array}{l}5.68 \\
(1.12)\end{array}$ \\
\hline & $\begin{array}{l}\text { I will volunteer to take on some responsibilities that are not my } \\
\text { own }\end{array}$ & $\begin{array}{l}4.88 \\
(1.43)\end{array}$ & $\begin{array}{l}4.93 \\
(1.36)\end{array}$ \\
\hline & $\begin{array}{l}\text { I will provide some good suggestions of the management } \\
\text { decisions for employers }\end{array}$ & $\begin{array}{l}4.63 \\
(1.55)\end{array}$ & $\begin{array}{l}4.45 \\
(1.52)\end{array}$ \\
\hline \multirow[t]{5}{*}{$\begin{array}{l}\text { Learning } \\
\text { performance }\end{array}$} & Total & $\begin{array}{l}16.10 \\
(3.47)\end{array}$ & $\begin{array}{l}16.06 \\
(3.28)\end{array}$ \\
\hline & $\begin{array}{l}\text { I attach great importance to learning and have a strong desire } \\
\text { to learn }\end{array}$ & $\begin{array}{l}5.54 \\
(1.24)\end{array}$ & $\begin{array}{l}5.37 \\
(1.21)\end{array}$ \\
\hline & $\begin{array}{l}\text { I have learned a lot of new working knowledge and skills } \\
\text { through various channels }\end{array}$ & $\begin{array}{l}5.30 \\
(1.28)\end{array}$ & $\begin{array}{l}5.32 \\
(1.15)\end{array}$ \\
\hline & $\begin{array}{l}\text { I have greatly improved my personal ability through study and } \\
\text { used it in my work }\end{array}$ & $\begin{array}{l}5.25 \\
(1.28)\end{array}$ & $\begin{array}{l}5.36 \\
(1.12)\end{array}$ \\
\hline & Total & $\begin{array}{l}63.66 \\
(12.01)\end{array}$ & $\begin{array}{l}64.16 \\
(10.99)\end{array}$ \\
\hline Options and & e: Never always: $1 \sim 7$ & & \\
\hline
\end{tabular}

In the one-way analysis of variance (Table 6), the job performance of both CSP and NCSP graduates showed statistically significant differences across medical schools, years of graduation, sexuality, and marital status. About job-related characteristics, job performance of graduates of CSP was also 
significantly different concerning whether becoming attending physicians, whether working in hometown district, and whether obtaining officially budgeted posts. Graduates of NCSP showed no difference in whether becoming attending physicians. 
Table 6

Mean score (SD) of the job performance scale by demographic and job-related variables Characteristics

$\operatorname{CSP}(n=1586) \quad \operatorname{NCSP}(n=568) \quad$ Overall $(n=2154)$

School

\begin{tabular}{llll} 
Qinghai & $61.03(13.27)$ & $63.11(10.85)$ & $61.55(12.58)$ \\
\hline Guangxi & $65.55(11.37)$ & $62.36(11.92)$ & $64.60(11.62)$ \\
\hline Jiujiang & $65.54(11.31)$ & $67.90(9.68)$ & $66.47(10.75)$ \\
\hline Gannan & $64.11(11.18)$ & $64.62(10.12)$ & $64.20(10.99)$ \\
\hline$P$ value & $<0.001$ & $<0.001$ & $<0.001$
\end{tabular}

Year of graduation

\begin{tabular}{|llll}
\hline 2015 & $66.47(12.42)$ & $66.13(10.64)$ & $66.33(11.71)$ \\
\hline 2016 & $65.62(11.82)$ & $65.85(8.89)$ & $65.68(11.07)$ \\
\hline 2017 & $63.15(12.35)$ & $61.79(12.45)$ & $62.82(12.38)$ \\
\hline 2018 & $62.08(11.19)$ & $64.21(10.95)$ & $62.55(11.16)$ \\
\hline 2019 & $62.07(11.78)$ & $61.27(11.29)$ & $61.92(11.68)$ \\
\hline$P$ value & $<0.001$ & $<0.001$ & $<0.001$ \\
\hline Demographic characteristics & & & \\
\hline Female & $62.55(11.25)$ & $62.92(11.06)$ & $62.65(11.19)$ \\
\hline Male & $64.70(12.59)$ & $65.50(10.78)$ & $64.90(12.17)$ \\
\hline$P$ value & $<0.001$ & 0.005 & $<0.001$ \\
\hline Not married & $62.89(11.85)$ & $63.58(11.38)$ & $63.07(11.73)$ \\
\hline Married & $65.36(12.25)$ & $65.43(10.01)$ & $65.38(11.66)$ \\
\hline$P$ value & $<0.001$ & 0.063 & $<0.001$
\end{tabular}

Current workplace

Public hospitals at county level and above $\quad 62.26(12.51) \quad 64.20(10.79) \quad 63.80(11.18)$

$\mathrm{CHC} \& \mathrm{THC}$

$63.79(11.97) \quad 62.58(13.01) \quad 63.78(11.98)$

Other

$64.44(11.00) \quad 64.22(12.75) \quad 64.25(12.38)$

$P$ value

0.372

0.881

0.957

Current job Information

Obtaining officially budgeted posts

$63.76(12.51) \quad 63.85(11.04)$

$63.77(11.90)$ 


\begin{tabular}{|llll|}
\hline Characteristics & CSP $(\mathbf{n = 1 5 8 6})$ & NCSP $(\mathbf{n = 5 6 8})$ & Overall $(\mathbf{n}=\mathbf{2 1 5 4})$ \\
\hline Not obtaining officially budgeted posts & $59.78(11.57)$ & $64.32(10.98)$ & $63.88(11.11)$ \\
\hline$P$ value & 0.038 & 0.632 & 0.864 \\
\hline Becoming Attending physicians & $66.82(12.76)$ & $65.501(9.19)$ & $66.80(12.68)$ \\
\hline Not becoming Attending physicians & $63.42(11.92)$ & $64.15(11.00)$ & $63.63(11.68)$ \\
\hline$P$ value & 0.004 & 0.863 & 0.005 \\
\hline Working in contract signing place & $63.74(11.99)$ & - & $63.74(11.99)$ \\
\hline Not working in contract signing place & $60.37(12.69)$ & - & $60.37(12.69)$ \\
\hline$P$ value & 0.101 & & 0.101 \\
\hline Working in hometown district & $64.86(11.56)$ & - & $64.86(11.56)$ \\
\hline Not working in hometown district & $61.91(12.45)$ & - & $61.91(12.45)$ \\
\hline$P$ value & $<0.001$ & & $<0.001$ \\
\hline
\end{tabular}

After controlling for the covariates listed in Table 6, we found that there was no significant difference between two types of graduates in job performance. This provides further evidence that although most medical graduates of CSP work in lower-level health facilities than NCSP, they do not show much difference in job performance. We then conducted five robustness checks to examine the consistency of the results. We replaced the dependent variable with the log of the job performance score (Table 7). The multivariable logistic regression was conducted (Appendix, table a1). We also replaced the dependent variable with task performance, contextual performance and learning performance (Appendix, table a2). Overall, the results were quite robust. No significant difference was detected between CSP and NCSP in a log scale, logistic regression, and of sub-dimensions for job performance.

However, CSP and NCSP graduates did show different patterns of influencing factors. For graduates of CSP, whether obtaining officially budgeted posts was a significant indicator of their job performance. Graduates who obtained officially budgeted posts scored $4.87(P<0.05)$ higher on average than those who did not obtain. Longer working years are also associated with higher job performance. Compared with 2015 CSP graduates, 2016-2019 graduates all showed significantly lower job performance $(\beta=-3.36$, $-3.53,-3.26$, respectively). Male and married graduates also showed significantly higher job performance $(\beta=1.85, P<0.01 ; \beta=1.54, P<0.05)$. 
Table 7

Multivariable regression on total job performance in CSP and NCSP graduates

\begin{tabular}{|c|c|c|c|c|c|c|c|c|}
\hline \multirow{3}{*}{$\begin{array}{l}\text { Variables } \\
\text { CSP }\end{array}$} & \multicolumn{8}{|c|}{$\beta$ coefficient (Standard error) } \\
\hline & \multicolumn{2}{|c|}{$\begin{array}{l}\text { Total } \\
\text { performance }\end{array}$} & \multicolumn{2}{|c|}{$\begin{array}{l}\text { Log total } \\
\text { performance }\end{array}$} & \multicolumn{2}{|c|}{$\begin{array}{l}\text { Total } \\
\text { performance in } \\
\text { CSP }\end{array}$} & \multicolumn{2}{|c|}{$\begin{array}{l}\text { Total } \\
\text { performance in } \\
\text { NCSP }\end{array}$} \\
\hline & -0.32 & $(1.16)$ & -0.01 & $(0.02)$ & & & & \\
\hline \multicolumn{9}{|l|}{$\begin{array}{l}\text { Reference: } \\
\text { Qinghai }\end{array}$} \\
\hline Guangxi & $3.03^{\star \star \star}$ & $(0.70)$ & $0.05^{\star \star \star}$ & $(0.01)$ & $3.87^{\star \star \star}$ & $(1.01)$ & -1.29 & $(1.21)$ \\
\hline Jiujiang & $3.95^{\star \star \star}$ & $(0.84)$ & $0.07^{\star \star \star}$ & $(0.02)$ & $3.23^{\star *}$ & $(1.12)$ & $4.39 * *$ & (1.33) \\
\hline Gannan & $2.61^{\star \star \star}$ & $(0.69)$ & $0.05^{\star \star \star}$ & $(0.01)$ & $2.77 \star \star$ & $(0.90)$ & 0.91 & $(1.36)$ \\
\hline \multicolumn{9}{|c|}{ Reference: graduated in 2015} \\
\hline $\begin{array}{l}\text { Graduated in } \\
2016\end{array}$ & -0.23 & $(0.93)$ & 0 & $(0.02)$ & -0.62 & $(1.33)$ & 0.28 & $(1.34)$ \\
\hline $\begin{array}{l}\text { Graduated in } \\
2017\end{array}$ & $-3.28^{\star \star \star}$ & $(0.95)$ & $-0.06 * \star$ & $(0.02)$ & $-3.46^{\star \star}$ & $(1.34)$ & $-3.39 *$ & $(1.39)$ \\
\hline $\begin{array}{l}\text { Graduated in } \\
2018\end{array}$ & $-2.72^{\star \star}$ & $(1.00)$ & $-0.04^{\star}$ & $(0.02)$ & $-3.53^{\star}$ & $(1.38)$ & -1.46 & $(1.53)$ \\
\hline $\begin{array}{l}\text { Graduated in } \\
2019\end{array}$ & $-3.10 * \star$ & $(1.01)$ & $-0.05^{\star}$ & $(0.02)$ & $-3.26^{\star}$ & $(1.38)$ & $-4.07 *$ & $(1.63)$ \\
\hline Male & $1.94^{\star \star \star}$ & $(0.50)$ & $0.03^{* \star}$ & $(0.01)$ & $1.85^{\star \star}$ & $(0.60)$ & $2.10 *$ & $(0.93)$ \\
\hline Married & $1.25^{\star}$ & $(0.59)$ & $0.02 *$ & $(0.01)$ & $1.54^{\star}$ & $(0.72)$ & 0.51 & $(1.05)$ \\
\hline $\begin{array}{l}\text { Officially } \\
\text { budgeted posts }\end{array}$ & 0.51 & $(0.92)$ & 0.01 & $(0.02)$ & $4.87 *$ & $(2.05)$ & -1.73 & $(0.99)$ \\
\hline $\begin{array}{l}\text { Becoming } \\
\text { Attending } \\
\text { physicians }\end{array}$ & -0.4 & $(1.36)$ & -0.01 & $(0.03)$ & -1.09 & $(1.63)$ & 1.77 & $(7.74)$ \\
\hline \multicolumn{9}{|c|}{ Reference: Working in public hospitals at county level and above } \\
\hline $\begin{array}{l}\text { Working in THC } \\
\& \mathrm{CHC}\end{array}$ & 0.19 & $(1.03)$ & 0.00 & $(0.02)$ & -0.80 & (1.17) & -3.60 & (3.27) \\
\hline $\begin{array}{l}\text { Working in other } \\
\text { places }\end{array}$ & 0.80 & $(1.63)$ & 0.01 & $(0.03)$ & 3.10 & $(4.05)$ & -0.45 & (1.68) \\
\hline
\end{tabular}

Note: (1) Source: Compulsory Services Program 2020 Wave. (2) $* \star \star p<0.001, * \star p<0.01, * p<0.05$; (3) Job income was measured in 1,000 CNY. (4) "Working in the contract signing place" and "Working in hometown district" were only for graduates of CSP. 


\begin{tabular}{|c|c|c|c|c|c|c|c|c|}
\hline \multirow{3}{*}{$\begin{array}{l}\text { Variables } \\
\text { Income of } \\
\text { current job }\end{array}$} & \multicolumn{8}{|c|}{$\beta$ coefficient (Standard error) } \\
\hline & \multicolumn{2}{|c|}{$\begin{array}{l}\text { Total } \\
\text { performance }\end{array}$} & \multicolumn{2}{|c|}{$\begin{array}{l}\text { Log total } \\
\text { performance }\end{array}$} & \multicolumn{2}{|c|}{$\begin{array}{l}\text { Total } \\
\text { performance in } \\
\text { CSP }\end{array}$} & \multicolumn{2}{|c|}{$\begin{array}{l}\text { Total } \\
\text { performance in } \\
\text { NCSP }\end{array}$} \\
\hline & 0.01 & $(0.07)$ & 0 & 0 & -0.04 & $(0.10)$ & 0.13 & $(0.09)$ \\
\hline $\begin{array}{l}\text { Working in } \\
\text { contract signing } \\
\text { place }\end{array}$ & - & - & - & - & 1.14 & $(2.12)$ & - & - \\
\hline $\begin{array}{l}\text { Working in } \\
\text { hometown } \\
\text { district }\end{array}$ & - & - & - & - & 1.29 & $(0.73)$ & - & - \\
\hline Constant & $61.92^{\star \star \star}$ & $(1.15)$ & 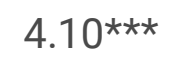 & $(0.02)$ & $56.69 * \star \star$ & $(2.79)$ & $63.68^{\star \star *}$ & $(1.51)$ \\
\hline R-sq & \multicolumn{2}{|l|}{0.047} & \multicolumn{2}{|l|}{0.040} & \multicolumn{2}{|l|}{0.058} & \multicolumn{2}{|l|}{0.084} \\
\hline $\mathrm{N}$ & \multicolumn{2}{|l|}{2154} & \multicolumn{2}{|l|}{2154} & \multicolumn{2}{|l|}{1586} & \multicolumn{2}{|l|}{568} \\
\hline $\begin{array}{l}\text { Note: (1) Source: } \\
\text { Job income was } \\
\text { hometown distric }\end{array}$ & $\begin{array}{l}\text { mpulsory } \\
\text { easured in } \\
\text { were only }\end{array}$ & $\begin{array}{l}\text { ervices } F \\
000 \text { CNY } \\
\text { r gradua }\end{array}$ & $\begin{array}{l}\text { ogram } 20 \\
\text { (4) “Work } \\
\text { es of CSP }\end{array}$ & $\begin{array}{l}0 \text { Wave } \\
\text { ig in the }\end{array}$ & $\begin{array}{l}\text { 2) } * * * p<0 \\
\text { ontract sig }\end{array}$ & $\begin{array}{l}\text { on, } * \star p \\
\text { ing plac }\end{array}$ & $\begin{array}{l}0.01, * \mathrm{p}< \\
\text { " and "Wor }\end{array}$ & $\begin{array}{l}\text {.05; (3) } \\
\text { ing in }\end{array}$ \\
\hline
\end{tabular}

The contracting strategy implemented by Qinghai is different from that of the other two provinces. Qinghai allows students to choose contract signing places according to their rank of college entrance examination score within the province, while Guangxi and Jiangxi require students to sign contracts with hometown counties in priority. If their hometowns cannot accept more CSP students or the candidates exceed the primary health institutions' recruiting capacity, students will be transferred to neighboring counties. Since Qinghai adopted different contracting strategy while the strategy is similar in the other two provinces, we separately controlled the different contract signing strategy in the regression for CSP (Table 8). We found that "working in hometown district" became significant, indicating that working in hometown might increase job performance $(\beta=1.48, P<0.05)$. 
Table 8

Multivariable regression on total job performance controlling for contracting strategy

\begin{tabular}{|c|c|c|}
\hline Variables & Bcoefficient & Standard error \\
\hline \multicolumn{3}{|l|}{ Reference: graduated in 2015} \\
\hline Graduated in 2016 & -0.59 & $(1.33)$ \\
\hline Graduated in 2017 & $-3.54^{\star \star}$ & $(1.33)$ \\
\hline Graduated in 2018 & $-3.59 * \star$ & $(1.37)$ \\
\hline Graduated in 2019 & $-3.27 *$ & $(1.37)$ \\
\hline Male & $1.79 * \star$ & $(0.60)$ \\
\hline Married & $1.47 *$ & $(0.71)$ \\
\hline Officially budgeted posts & $4.73^{\star}$ & (2.05) \\
\hline Becoming Attending physicians & -0.93 & $(1.62)$ \\
\hline \multicolumn{3}{|c|}{ Reference: Working in public hospitals at county level and above } \\
\hline Working in $\mathrm{THC} \& \mathrm{CHC}$ & -0.73 & $(1.17)$ \\
\hline Working in other places & 3.20 & $(4.05)$ \\
\hline Income of current job & -0.04 & $(0.10)$ \\
\hline Working in contract signing place & 1.35 & $(2.11)$ \\
\hline Working in hometown district & $1.48^{\star}$ & $(0.70)$ \\
\hline Contracting strategy & $-3.15^{\star \star \star}$ & $(0.82)$ \\
\hline Constant & $59.71 * \star \star$ & $(2.71)$ \\
\hline R-sq & 0.057 & \\
\hline Observations & 1586 & \\
\hline \multicolumn{3}{|c|}{ 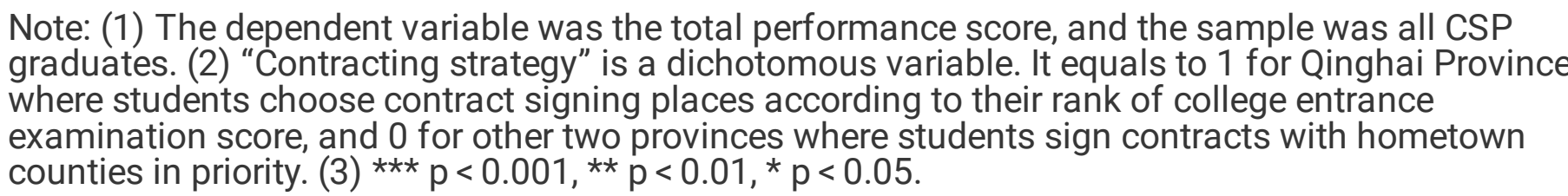 } \\
\hline
\end{tabular}

\section{Discussion}

This study tried to evaluate the job performance of medical graduates trained by CSP, while most existing studies focused on attraction and retention [20, 41-44]. We found that CSP graduates have demonstrated good job performance in primary health settings in China. Although CSP students were 
recruited with lower college entrance examination scores, the pass rate of NLME and self-reported job performance scale both have suggested that CSP graduates exhibited as good job performance as NCSP peers who performed better in college entrance examination.

International CSP experiences confirmed that students improved academic performance during school. In Japan, Matsumoto and colleagues found that students increased their academic standing throughout undergraduate education [45]. In US, Rabinowitz also found that although students had lower test scores than non-program peers of the same medical schools when matriculated, the difference decreased during medical education and disappeared when they graduated [46]. We further confirmed that such positive changes persist after students graduate and continue after they begin practicing medicine. Our study suggests that CSP graduates are well-trained and have the competency to become qualified workforces in primary health systems.

Currently, most rural primary health workers in China have only received limited medical training and lower qualification. In 2019, $82.6 \%$ health professionals at township health center received medical education for only 3 years or even less. This contributes to the low trust of rural residents on PHC service quality [47]. As a result, most rural residents bypass the $\mathrm{PHC}$ services and directly go to higher hospital for even minor conditions [48]. Increasing the supply of physicians and their job performance/service quality are equally important for China's rural health system. Without CSP, those well-trained five-year medical graduates would not choose to work in rural or remote areas due to lower income, limited career opportunities and other limitations. Since the college entrance scores of CSP students on matriculation are lower than that of NCSP students. One would concern whether their job performance and the quality of health services they provide is also lower than that of NCSP. However, our results suggest that there is no significant difference between the two types of graduates, indicating that after five-year undergraduate education, the quality and competency of CSP graduates can been guaranteed. CSP students receive standardized residency training as NCSP, which may also explain this improvement. Through this comprehensive training, CSP graduates could increase the accessibility of equal-quality or high-quality services in rural areas, and also help to address the deficit and maldistribution of health workforce and improve health equity in rural and remote areas.

As for the influencing factors, officially budgeted posts and working in hometown have positive influence on job performance. Officially budgeted posts can increase employees' recognition towards the organization and maintain close contact between employees and employers [49]. The national policies promise to provide officially budgeted posts for CSP graduates, which is also the primary attraction for students' recruitment. However, due to limitations in decentralization, THC cannot fully decide their officially budgeted posts when recruiting new employees [28]. The posts may hang for a long time, discouraging CSP graduates' working enthusiasm, especially for newly graduated students, who showed lower job performance compared to those who have worked for a longer period. The first few years of working in rural areas may be critical for career development and future retention. Before officially budgeted posts fulfill, the health facilities and local governments should provide more support to enhance job performance to secure service quality, as well as increase retention in the long run. 
Working in hometown could also increase the job performance of CSP graduates. "Whether working in hometown" was insignificant in multivariable regression, but became significant again when we controlled for the contracting strategies. In Qinghai, the contract signing places are not related to students' hometown necessarily, because the top-ranked students will give priority to developed areas with lower altitude, even if they come from higher-altitude and less developed areas. Signing contracts with hometowns is not always a desired option. As a result, low-ranked students may be transferred to less desirable working places after graduation. Their job performance may decrease. While in Jiangxi and Guangxi, as stipulated by the policies, students' primary contract signing places are their hometowns. Some studies have shown that hometown recruiting can increase the probability of retention in rural health institutions [16]. Students who work in their hometowns were more satisfied with CSP policies. However, there is limited evidence about which way of signing contracts will predict better job performance outcome. Our results suggest that signing contracts with hometown might have positive impact on students' job performance, which might increase their retention in the long run.

This study has several limitations. First, this study used self-reported data to measure the job performance. It might be subject to self-report bias to distinguish the two types of medical graduates. Second, the attrition of NCSP graduates is higher than that of CSP. NCSP graduates have more diversified employment status, making them difficult to follow up. The different attrition rates might bring bias to the results. Third, due to the pandemic of COVID-19, the NMLE in 2020 has been delayed. Most students graduated in 2019 had not taken the exam by the time when we conducted the follow-up survey. We can only obtain the pass rate of the NMLE for graduates in $2015,2016,2017$, and 2018 , which might affect the results.

In conclusion, CSP graduates have demonstrated good job performance, showing the potential competence to provide high-quality care to a large population in rural areas in China. Whether working in hometowns and obtaining officially budgeted posts can have positive influence on job performance and may influence future retention. Early career support can have a crucial influence on graduates, especially the first few years after graduation. Early career support should be provided to allow them to maintain high job performance for better service quality and retention.

\section{Abbreviations}

CSP: Compulsory services program; NCSP: non-compulsory services program; CNY: Chinese yuan; NMLE: National Medical Licensing Examinations; GP: general practitioner; $\mathrm{CHC}$ : community health center; THC: township health center.

\section{Declarations}

\section{Acknowledgements}


The authors want to thank Qinghai University, Jiujiang University, Gannan Medical University, and Guangxi Medical University for their support in the establishment of the cohorts.

\section{Ethics approval and consent to participate}

The study has been approved by the Institutional Review Board of Peking University (PU IRB). Informed consent was obtained from all participants prior to questionnaire administration.

\section{Availability of data and materials}

The data used and/or analyzed during the study are available from the corresponding author on reasonable request.

\section{Competing interests}

The authors declare that they have no competing interests.

\section{Funding}

The Cohort Study of Medical Graduates with Compulsory Services in Rural Areas Studies was funded by China Medical Board (CMB). The funder had no role in study design, data collection, data analysis, data interpretation, or writing of the report.

\section{Authors' contributions}

ML drafted the manuscript, and conducted the data analysis and interpretation. BZ, TW, DH collected and managed the data. XL and ZW designed the study, managed the data, and made critical interpretations and revisions on some intellectual contents of the article. All authors gave final approval for the final version to be published.

\section{Consent for publication:}

Not applicable.

\section{Author details}

Mingyue Li, Ziyue Wang, Baisong Zhang, Tiantian Wei, Dan Hu, Xiaoyun Liu: China Center for Health Development Studies, Peking University, 38 Xueyuan Road, Haidian District, Beijing, 100191, China 
Mingyue Li, Baisong Zhang \& Tiantian Wei: School of Public Health, Peking University Health Science Center, 38 Xueyuan Road, Haidian District, Beijing, 100191, China

\section{References}

1. WHO: Increasing access to health workers in remote and rural areas through improved retention. World Health Organization

2. Weller B: Guidelines: Incentives for Health Professionals. Global Health Workforce Alliance

3. Rowe AK, de Savigny D, Lanata CF, Victora CG: How can we achieve and maintain high-quality performance of health workers in low-resource settings? The Lancet 2005, 366:1026-1035.

4. Aluttis $\mathrm{C}$, Bishaw T, Frank MW: The workforce for health in a globalized context - global shortages and international migration. Global Health Action 2014, 7.

5. Ahmed SM, Hossain MA, Rajachowdhury AM, Bhuiya AU: The health workforce crisis in Bangladesh: shortage, inappropriate skill-mix and inequitable distribution. Hum Resour Health 2011, 9:3.

6. Dieleman M, Kane S, Zwanikken P, Gerretsen B: Realist review and synthesis of retention studies for health workers in rural and remote areas. Journal of the American College of Surgeons 2010, 216:353-362.

7. Humphreys J, Wakerman J, Pashen D, Buykx P: Retention strategies and incentives for health workers in rural and remote areas: what works? 2017.

8. Richards HM, Farmer J, Selvaraj S: Sustaining the rural primary healthcare workforce: survey of healthcare professionals in the Scottish Highlands. Rural Remote Health 2005, 5:365.

9. Dywili S, Bonner A, Anderson J, O'Brien L: Experience of overseas-trained health professionals in rural and remote areas of destination countries: A literature review. Australian Journal of Rural Health 2012, 20:175-184.

10. Wilson NW, Couper ID, De Vries E, Reid S, Fish T, Marais BJ: A critical review of interventions to redress the inequitable distribution of healthcare professionals to rural and remote areas. Rural Remote Health 2009, 9:1060.

11. Belaid L, Dagenais C, Moha M, Ridde V: Understanding the factors affecting the attraction and retention of health professionals in rural and remote areas: a mixed-method study in Niger. Hum Resour Health 2017, 15:60.

12. Mbemba G, Gagnon MP, Paré G, Côté J: Interventions for supporting nurse retention in rural and remote areas: an umbrella review. Hum Resour Health 2013, 11:44.

13. Morell AL, Kiem S, Millsteed MA, Pollice A: Attraction, recruitment and distribution of health professionals in rural and remote Australia: early results of the Rural Health Professionals Program. Hum Resour Health 2014, 12:15.

14. Frehywot S, Mullan F, Payne PW, Ross H: Compulsory service programmes for recruiting health workers in remote and rural areas: do they work? Bull World Health Organ 2010, 88:364-370. 
15. Stenger J, Cashman SB, Savageau JA: The primary care physician workforce in Massachusetts: implications for the workforce in rural, small town America. J Rural Health 2008, 24:375-383.

16. Matsumoto M, Inoue K, Kajii E: Policy implications of a financial incentive programme to retain a physician workforce in underserved Japanese rural areas. Soc Sci Med 2010, 71:667-671.

17. Dolea C, Stormont L, Braichet JM: Evaluated strategies to increase attraction and retention of health workers in remote and rural areas. Bull World Health Organ 2010, 88:379-385.

18. Li H, Yuan B, Wang D, Meng Q: Motivating factors on performance of primary care workers in China: a systematic review and meta-analysis. BMJ Open 2019, 9:e028619.

19. Yuan B, Meng Q, Song K, Hou Z: Preference for working motivation and its influencing factors among rural health workers (in Chinese). Chinese Journal of Public Health 2012, 28.

20. Antonio CT, Guevarra JP, Medina PN, Avelino MD, Agbon AG, Sepe DC, Bardelosa DD, Cengca MM, Ting ML, Lara AB: Components of compulsory service program for health professionals in low- and middle-income countries: a scoping review. Perspect Public Health 2020, 140:54-61.

21. Hatcher AM, Onah M, Kornik S, Peacocke J, Reid S: Placement, support, and retention of health professionals: national, cross-sectional findings from medical and dental community service officers in South Africa. Hum Resour Health 2014, 12:14.

22. Lehmann U, Dieleman $M$, Martineau T: Staffing remote rural areas in middle- and low-income countries: a literature review of attraction and retention. BMC Health Serv Res 2008, 8:19.

23. Sánchez Del Hierro G, Remmen R, Verhoeven V, Van Royen P, Hendrickx K: Are recent graduates enough prepared to perform obstetric skills in their rural and compulsory year? A study from Ecuador. BMJ Open 2014, 4:e005759.

24. Yip W, Fu H, Chen AT, Zhai T, Jian W, Xu R, Pan J, Hu M, Zhou Z, Chen Q, et al: 10 years of health-care reform in China: progress and gaps in Universal Health Coverage. The Lancet 2019, 394:1192-1204.

25. Ma X, Wang H, Yang L, Shi L, Liu X: Realigning the incentive system for China's primary healthcare providers. Bmj 2019, 365:12406.

26. People's Republic of China: Notice on Issuing the Implementation Opinions on Medical Students of Compulsory Services Programs for Rural Areas. 2010.

27. Zhang Z, Zhang C, Huang M, Liu M, Zuo Y, Yan B, Liu X: Two-year follow-up analysis of targeted admission medical graduates in rural areas (in Chinese). Chinese Journal of Health Policy 2018, 11:34-39.

28. Zhang C, Chen C, Zhang Z, Wang W, Hu D, Huang M, Yan B, Yu J, Tian J, Liu X: Study on the current employment situation of targeted admission medical graduates in rural areas (in Chinese). Chinese Journal of Health Policy 2017, 10:27-33.

29. Wang Z, Hu D, B. Z, M. H, M. L, Y. Z, Z. J, Liu X: Analysis on the targeted admission medical graduates and their work preference in rural areas: A three-year survey conducted in four medical schools (in Chinese). Chinese Journal of Health Policy 2020, 13:57-62. 
30. Standing Committee of the National People's Congress of China: Law on Practicing Doctors of the People's Republic of China. 1999.

31. Han X, Li X, Cheng L, Wu Z, Zhu J: Performance of China's new medical licensing examination for rural general practice. BMC Med Educ 2020, 20:314.

32. Koopmans L, Bernaards CM, Hildebrandt VH, Schaufeli WB, de Vet Henrica CW, van der Beek AJ: Conceptual frameworks of individual work performance: a systematic review. J Occup Environ Med 2011, 53:856-866.

33. Motowildo SJ, Borman WC, Schmit MJ: A Theory of Individual Differences in Task and Contextual Performance. Human Performance 1997, 10:71-83.

34. Han Y, Liao J, Long L: Construction and Empirical Research of employee job Performance structure Model. Hubei Changjiang Publishing Group; 2007.

35. Kirton MJ: Adaptors and innovators-Why new initiatives get blocked. Long Range Planning 1984, 17:137-143.

36. Belbin RM: Team Roles at Work. Butterworth-Heinemann (Oxford and Boston)

37. Zhao S: Study on the current situation and relationship of work attitude and performance of health techinical personnel in township health centers of three provinces in China (in Chinese). PhD dissertation. Shandong University, 2015.

38. Wei Y, Wei X: Relationship between leadership of general team leaders and work performance: a structural equation model analysis (in Chinese). Chinese General Practice 2019, 22:16-20.

39. Wei $Y$, Wei $X$ : Study on the influencing factors of work performance of general practitioner team members in Shanghai (in Chinese). Chinese General Practice 2019, 22:22-25.

40. Yao X, Wang X, Rem X, Zhai P, Hao Q: Effect of post competency on the work performance of rural doctors: a study in Shanxi province (in Chinese). Chinese Jounal of General Practice 2021, 20:61-66.

41. Sidibé CS, Touré O, Broerse JEW, Dieleman M: Rural pipeline and willingness to work in rural areas: Mixed method study on students in midwifery and obstetric nursing in Mali. PLoS One 2019, 14:e0222266.

42. Kroezen M, Dussault G, Craveiro I, Dieleman M, Jansen C, Buchan J, Barriball L, Rafferty AM, Bremner J, Sermeus W: Recruitment and retention of health professionals across Europe: A literature review and multiple case study research. Health Policy 2015, 119:1517-1528.

43. Carson DB, Schoo A, Berggren P: The 'rural pipeline' and retention of rural health professionals in Europe's northern peripheries. Health Policy 2015, 119:1550-1556.

44. Pagaiya N, Kongkam L, Sriratana S: Rural retention of doctors graduating from the rural medical education project to increase rural doctors in Thailand: a cohort study. Hum Resour Health 2015, 13:10.

45. Matsumoto M, Inoue K, Kajii E: Characteristics of medical students with rural origin: implications for selective admission policies. Health Policy 2008, 87:194-202. 
46. Rabinowitz HK: A program to recruit and educate medical students to practice family medicine in underserved areas. Jama 1983, 249:1038-1041.

47. National Health Commission: China National Health Statistical Yearbook 2020. Peking Union Medical College Press; 2020.

48. Meng Q, Yin D, Mills A, Abbasi K: China's encouraging commitment to health. Bmj 2019, 365:14178.

49. Lewis M, Pettersson G: Governance in health care delivery: raising performance. The World Bank; 2009.

\section{Figures}

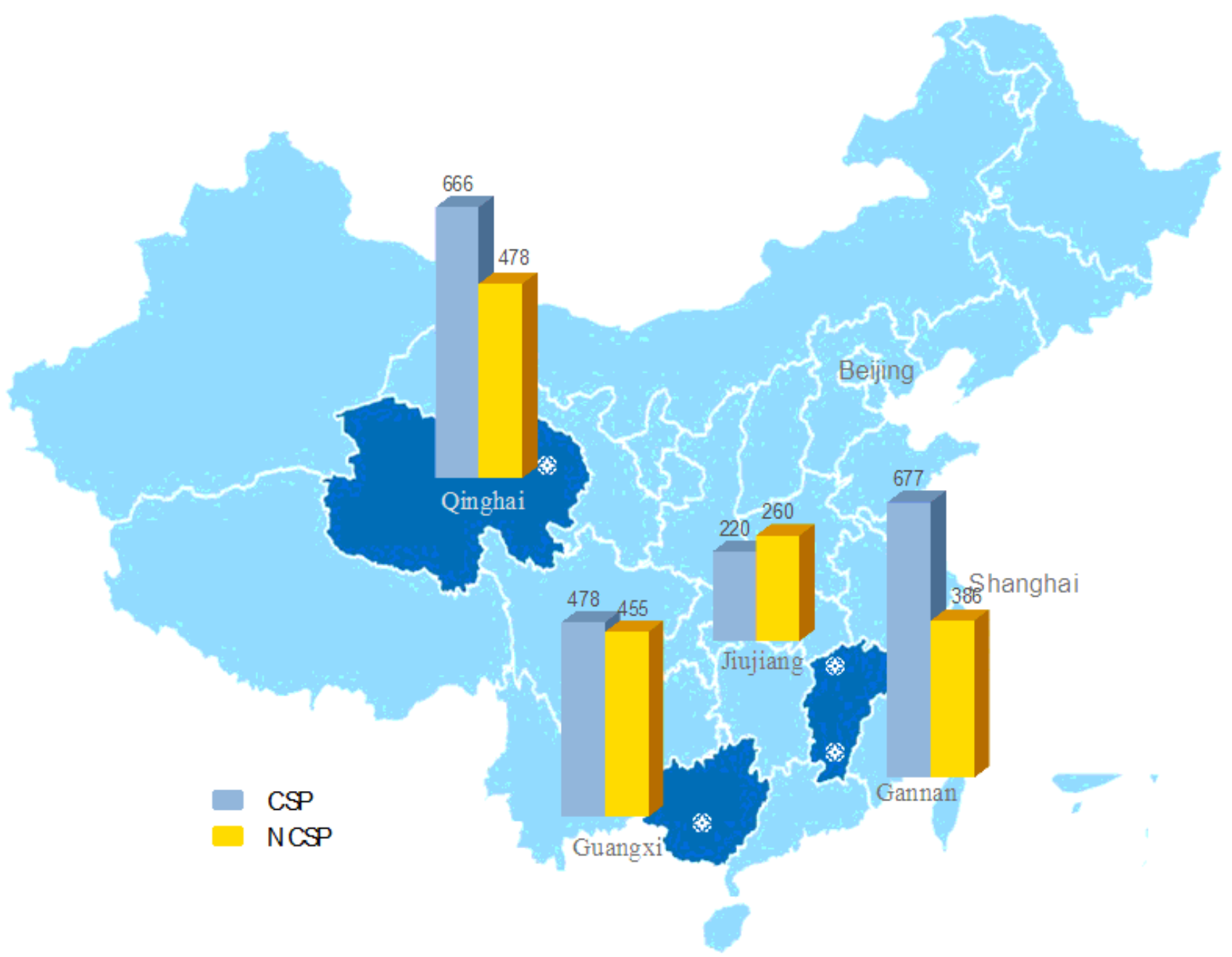

\section{Figure 1}

Locations of four medical universities in China and initial cohort size Note: The designations employed and the presentation of the material on this map do not imply the expression of any opinion whatsoever on the part of Research Square concerning the legal status of any country, territory, city or area or of its 
authorities, or concerning the delimitation of its frontiers or boundaries. This map has been provided by the authors.

\section{Supplementary Files}

This is a list of supplementary files associated with this preprint. Click to download.

- Appendix.docx 